\title{
Variability in gelatinase expression in the walls of vessels used as aortocoronary conduits may impact long-term graft patency
}

\author{
Bartłomiej Perek ${ }^{1}$, Katarzyna Kowalska², Michał Nowicki², Bartosz Kempisty2, 3 , \\ Mateusz Puślecki ${ }^{1,4}$, Maurycy Jankowski ${ }^{3}$, Marek Jemielity ${ }^{1}$, Agnieszka Malińska ${ }^{2}$ \\ 'Department of Cardiac Surgery and Transplantology, Poznan University of Medical Sciences, Poznan, Poland \\ 2Department of Histology and Embryology, Poznan University of Medical Sciences, Poznan, Poland \\ ${ }^{3}$ Department of Anatomy, Poznan University of Medical Sciences, Poznan, Poland \\ ${ }^{4}$ Department of Medical Rescue, Poznan University of Medical Sciences, Poznan, Poland
}

\begin{abstract}
A bstract
Background: An imbalance between the activity of matrix metalloproteinases (MMPs), particularly gelatinases, and tissue inhibitors of metalloproteinases (TIMPs) is considered as one of the mechanisms leading to aortocoronary graft failure.

Aim: We aimed to assess the variability in gelatinase expression in the walls of aortocoronary conduits and to evaluate its impact on coronary artery bypass grafting (CABG) outcomes.

Methods: The study included 101 consecutive patients (61 men and 40 women) who underwent CABG. An immunohistochemical analysis of MMP-2, MMP-9, TIMP-1, and TIMP-2 expression was performed on the cross-sections of the internal thoracic artery (ITA), radial artery (RA), and saphenous vein (SV). The histological findings were compared between patients with SV graft disease (SVGD[+] group) and those without occlusions in the SV (SVGD[-] group).

Results: The median MMP and TIMP expression was the weakest in the ITA wall. MMP expression was comparable between the RA and SV cross-sections, whereas TIMP expression was stronger in the RA than in the SV wall ( $<<0.05)$. In most SV segments, but not in the arteries, immunostaining intensity for MMP was comparable to or stronger than for TIMPs. In the veins harvested from the SVGD(+) group, MMP-2 and MMP-9 tissue expression was more pronounced than in the SVGD(-) group. TIMP levels were comparable between groups.

Conclusions: Imbalance in the metalloproteinase-to-inhibitor tissue expression in the vessel wall might predispose to graft failure. A stronger expression of TIMPs than MMPs in the arterial grafts might explain favourable long-term outcomes.

Key words: coronary artery bypass graft, graft disease, metalloproteinases, tissue inhibitors of metalloproteinases, outcomes

Kardiol Pol 2019; 77, 2: 217-224
\end{abstract}

\section{INTRODUCTION}

Coronary artery bypass grafting $(\mathrm{CABG})$ is a method of choice in the treatment of patients with extensive coronary artery disease (CAD) [1]. The late angiographic and clinical outcomes depend both on the progression of CAD in the native arteries and on the patency of the implanted aortocoronary conduits $[2,3]$. Although transplantation of the left internal thoracic artery (ITA) to the left anterior descending artery has been considered as a gold standard for many years, other arterial and saphenous vein (SV) segments have been extensively used in CABG procedures [4, 5]. Long-term outcomes of the other grafts are often conflicting but more reports favour the radial artery (RA) as the second-best graft [3, 6].

Investigators have been searching for possible factors that might determine the unfavourable outcome of the implanted conduits [7, 8]. So far, numerous cell lines and bioactive molecules have been implicated. Among others, two matrix metalloproteinases (MMPs), namely MMP-2 and MMP-9, also known as gelatinases, have been shown to be involved in extracellular matrix (ECM) degradation, which is

\section{Address for correspondence:}

Bartłomiej Perek, MD, PhD, Department of Cardiac Surgery and Transplantology, Poznan University of Medical Sciences, ul. Długa 1/2, 61-848 Poznań, Poland, tel: +48 6185492 10, e-mail: bperek@ump.edu.pl 
Table 1. Baseline demographic and clinical characteristics of patients with and without saphenous vein graft disease (SVGD)

\begin{tabular}{|c|c|c|c|c|}
\hline Variables & $\begin{array}{c}\text { All } \\
(n=101)\end{array}$ & $\begin{array}{l}\operatorname{SVDG}(+)^{*} \\
(n=24)\end{array}$ & $\begin{array}{l}\text { SVGD(-)* } \\
(n=72)\end{array}$ & $\begin{array}{l}\text { p (SVGD }[+] \\
\text { vs. SVGD[-]) }\end{array}$ \\
\hline Age [years] & $64.2 \pm 9.8$ & $63.4 \pm 10.4$ & $64.5 \pm 9.2$ & NS \\
\hline \multicolumn{5}{|l|}{ Clinical data: } \\
\hline Obesity (BMI > 30 kg/m²) & $40(39.6)$ & $10(41.6)$ & $28(38.9)$ & NS \\
\hline Arterial hypertension & $66(65.3)$ & $20(83.3)$ & $43(59.7)$ & 0.035 \\
\hline Insulin-treated diabetes & $26(25.7)$ & $8(33.3)$ & $15(20.8)$ & NS \\
\hline Hyperlipidaemia & $41(40.6)$ & $12(50.0)$ & $29(40.3)$ & NS \\
\hline Renal failure ${ }^{1}$ & $13(12.9)$ & $3(12.5)$ & $10(13.9)$ & NS \\
\hline Peripheral vascular disease & $25(24.8)$ & $6(25.0)$ & $16(22.2)$ & NS \\
\hline Neurological events² & $9(8.9)$ & $2(8.3)$ & $5(6.9)$ & NS \\
\hline Active smoking $^{3}$ & $51(50.5)$ & $15(62.5)$ & $31(43.1)$ & NS \\
\hline \multicolumn{5}{|c|}{ Preoperative coronary angiography: } \\
\hline Left main disease & $23(22.8)$ & $5(20.8)$ & $17(23.6)$ & NS \\
\hline Two-vessel disease & $15(14.9)$ & $3(12.5)$ & $11(15.3)$ & NS \\
\hline Three-vessel disease & $63(62.3)$ & $16(66.7)$ & $44(61.1)$ & NS \\
\hline
\end{tabular}

Categorical variables are presented as a number (percentage), and continuous variables as a mean \pm standard deviation. BMI — body mass index; NS - non-significant

*The sum of SVGD(-) and SVGD(+) patients is not 101 because five patients were lost to follow-up.

'Diagnosed if a glomerular filtration rate was below $60 \mathrm{~mL} / \mathrm{min} / 1.73 \mathrm{~m}{ }^{2} ;{ }^{2}$ Included both transient ischaemic attacks and strokes; ${ }^{3}$ The term

"active smokers" comprises active smokers and the individuals who had given up smoking within one year before surgery

of paramount importance in the promotion of cell migration within the vessel wall, followed by neointima formation and atherosclerosis $[9,10]$.

The biological activity of these gelatinases is physiologically balanced by their tissue inhibitors (tissue inhibitors of metalloproteinases [TIMPs]). There is mounting evidence pointing towards an imbalance between MMP and TIMP activity, rather than the expression of gelatinases themselves, as the determinant of ultrastructural changes within the vessel wall [11].

Considering the above, we assessed the variability in gelatinase expression in the walls of the vessels used as aortocoronary conduits, before the CABG procedure, and evaluated whether these differences may have clinical significance.

\section{METHODS \\ Patients}

This study included 101 consecutive patients (61 men and 40 women) at a mean age of $64.2 \pm 9.8$ years (range, $42-$ -84 years), who underwent non-emergent CABG. Patients were referred for surgery on the basis of coronary angiography. At the end of the follow-up, they were classified into two subgroups: one with SV graft disease (SVGD[+]) and the other without SV graft occlusions (SVGD[-]). Selected baseline data are summarised in Table 1.

The study protocol was approved by the Bioethical Committee of the Poznan University of Medical Sciences (1207/08). All patients gave written informed consent to participate in the study.

\section{Biological material procurement and preparation}

During off-pump CABG procedures performed mostly (97 operations; $96.3 \%$ ) by surgeons with at least five years of experience in coronary surgery, surplus segments of the vessels used as aortocoronary grafts were obtained for ultrastructural studies. In all cases, the most distal segments, at least one centimetre in length, were harvested. Both arteries (ITA and RA) were dissected free as pedicled grafts, whereas the SV was obtained in the standard manner, applying a full skin incision over its entire course. To minimise possible injury to the vessels, surgeons avoided touching (no-touch technique), excessive manipulation, and dilation or use of high-energy electrocautery. As a result, 230 vessel segments (101 ITA, 101 SV, and 28 RA segments) were saved for histological analysis.

The segments were subjected to a macroscopic inspection for any abnormalities, following which they were carefully rinsed with $0.9 \% \mathrm{NaCl}$ at room temperature, slightly dilated, and immersed in the freshly prepared Boiun solution for light microscopic studies. The subsequent steps of preparation for light microscopic examinations were described in detail previously [12]. All immunohistochemical analyses were performed with Dako REAL EnVision Detection System, Peroxidase/DAB, Rabbit/Mouse, K5007 (Dako, Copenhagen, Denmark) and were conducted according to the standard procedure [12]. The following mouse monoclonal antibodies were used: anti-MMP-2 (dilution 1:50; NB200-114), anti-MMP-9 (dilution 1:250; NB100-78556), anti-TIMP-1 (1:150; NBP1-96554), 
Table 2. Results of matrix metalloproteinase (MMP) expression analysis

\begin{tabular}{|c|c|c|c|c|}
\hline & ITA $(n=101)$ & $\mathrm{RA}(\mathrm{n}=28)$ & SV $(n=101)$ & Statistical analysis $* * *$ \\
\hline \multicolumn{5}{|c|}{ Tissue expression of proteins (IRS score):* } \\
\hline MMP-2 & $1(0 ; 2)$ & $4(2 ; 4)$ & $4(2 ; 4)$ & $\mathrm{ITA}<\mathrm{RA}=\mathrm{SV}$ \\
\hline MMP-9 & $2(1 ; 4)$ & $3.5(2 ; 4)$ & $4(2 ; 4)$ & $\mathrm{ITA}<\mathrm{RA}=\mathrm{SV}$ \\
\hline TIMP-1 & $2(1 ; 2)$ & $4(2 ; 4)$ & $2(2 ; 4)$ & $\mathrm{ITA}<\mathrm{SV}<\mathrm{RA}$ \\
\hline TIMP-2 & $4(4 ; 4.5)$ & $6(6 ; 6.5)$ & $6(4 ; 6)$ & $\mathrm{ITA}<\mathrm{RA}<\mathrm{SV}$ \\
\hline \multicolumn{5}{|c|}{ Tissue expression of MMPs vs. TIMPs: ** } \\
\hline MMP-2 > TIMP-1 & $11(10.9)$ & $5(19.2)$ & $53(52.5)$ & $\mathrm{ITA}<\mathrm{RA}=\mathrm{SV}$ \\
\hline MMP-2 > TIMP-2 & $0(0)$ & $1(3.8)$ & $38(37.6)$ & NS \\
\hline MMP-9 > TIMP-1 & $29(28.7)$ & $7(26.9)$ & $23(22.8)$ & $\mathrm{ITA}=\mathrm{RA}<\mathrm{SV}$ \\
\hline MMP-9 > TIMP-2 & $0(0)$ & $0(0)$ & $7(6.9)$ & NS \\
\hline
\end{tabular}

*Data (IRS according to Remmele score) are expressed as medians $\left(25^{\text {th }} ; 75^{\text {th }}\right.$ percentile) or ** numbers [n] with percentage; *** " $<$ " if $p$ value $<0.5$; IRS — immunoreactivity score; ITA — internal thoracic artery; NS — non-significant; RA — radial artery; SV — saphenous vein; TIMP — tissue inhibitor of metalloproteinase

and anti-TIMP-2 (dilution 1:200; NB110-61001) (all Novus Biologicals, Littleton, CO, USA). The peroxidase reaction was conducted with the use of diaminobenzidine.

\section{Immunohistochemical analysis of protein expression}

Immunohistochemical reactions were analysed using an Olympus BX 50 light microscope (OLYMPUS Optical Europe, Germany), equipped with a Mirax-Midi scanner (Carl Zeiss Microlmaging $\mathrm{GmbH}$, Germany) and compatible with image visualisation software (Pannoramic Viewer ver. 1.15.4.; 3DHISTECH Ltd., Budapest, Hungary).

The intensity of the cytoplasmic expression of proteins was blindly assessed by two experienced histologists on 10 representative vessel cross-sections, using the semiquantitative immunoreactive score (IRS) according to Remmele and Stegner [13]. This scale takes the percentage of positive cells (scale from 0 to 4 ) and the intensity of the colour reaction (scale from 0 to 3 ) into account, with the final score, ranging from 0 to 12, being the sum of points given for individual traits. According to the IRS, the expression of cytoplasmic proteins was considered as negative (IRS 0-1), weak (IRS 2-3), moderate (IRS 4-6), or strong (IRS 8-12).

\section{Postoperative outcomes}

All patients were systematically followed in an outpatient clinic after discharge. Special attention was paid to symptoms of CAD progression. The composite primary clinical endpoint comprised cardiac-related death, acute coronary syndrome, or the necessity to undergo coronary angiography due to progression of angina, according to the Canadian Cardiovascular Society score. No follow-up angiography was performed in asymptomatic individuals, except patients with diabetes, who had control coronary angiography irrespective of CAD symptoms. Patients who reached the primary endpoint and had occlusions in the SV grafts were assigned to the SVGD $(+)$ group, whereas the remaining patients were assigned to the SVGD(-) group. The histological findings for the graft segments were compared between groups.

\section{Statistical analysis}

Continuous variables were checked for normality with the Shapiro-Wilk W test. If they met the criteria of normal distribution, they were expressed as means with standard deviations. Categorical variables (IRS) were presented as medians with the $25^{\text {th }}$ and $75^{\text {th }}$ percentiles. Dichotomous data were expressed as absolute numbers (n) with percentages (\%), and, similarly to the medians, they were compared using the Kruskal-Wallis test and, if necessary, with multiple comparisons of mean ranks for all groups.

Vascular segments harvested from the $\operatorname{SVGD}(+)$ and SVGD (-) groups were compared, and the results were analysed using the unpaired Student t test or the nonparametric Mann-Witney $U$ test. Baseline clinical data (as dichotomous variables) of SVGD(-) and SVGD(+) groups were compared using the $\chi^{2}$ test. A p-value of less than 0.05 was considered significant. The analysis was performed with the use of the Statistica 10.0 software for Windows (StatSoft, Inc., Tulsa, OK, USA).

\section{RESULTS}

\section{Tissue expression of gelatinases}

The median MMP-2 and MMP-9 expression in vessel cross-sections differed significantly between the types of vessels (Table 2). The weakest expression was observed in ITA walls, whereas the RA and SV segments presented comparable results (Fig. 1).

No MMP-9 expression (IRS 0-1) was noted in the majority of ITA cross-sections $(66 ; 65.3 \%)$, as com- 

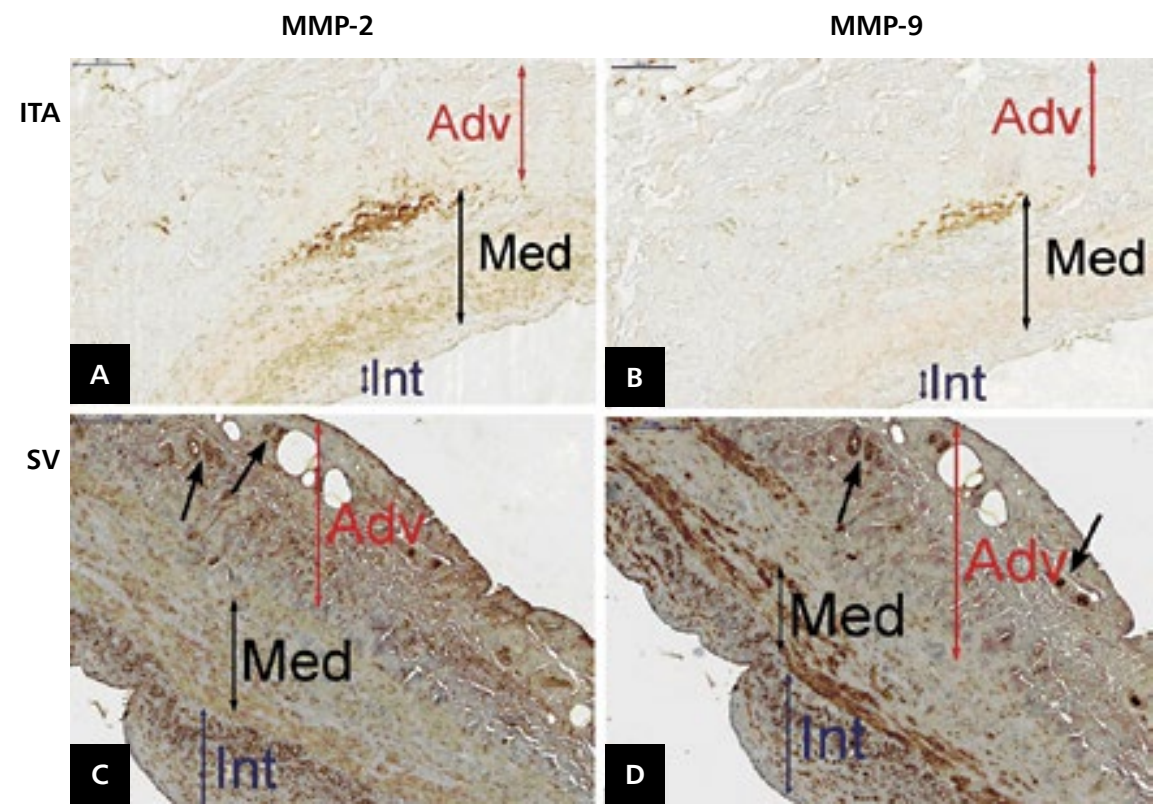

Figure 1. Matrix metalloproteinase (MMP) expression in the arterial wall. Intensity of immunostaining for MMP-2 (A, C) and MMP-9 (B, D) observed in the tunica media (Med) of the internal thoracic artery (ITA) cross-sections was much weaker than in saphenous vein (SV) wall. MMP-2-positive cells were also found in the wall of the vasa vasorum (arrows) on SV cross-sections; Adv — tunica adventitia; Int — tunica intima
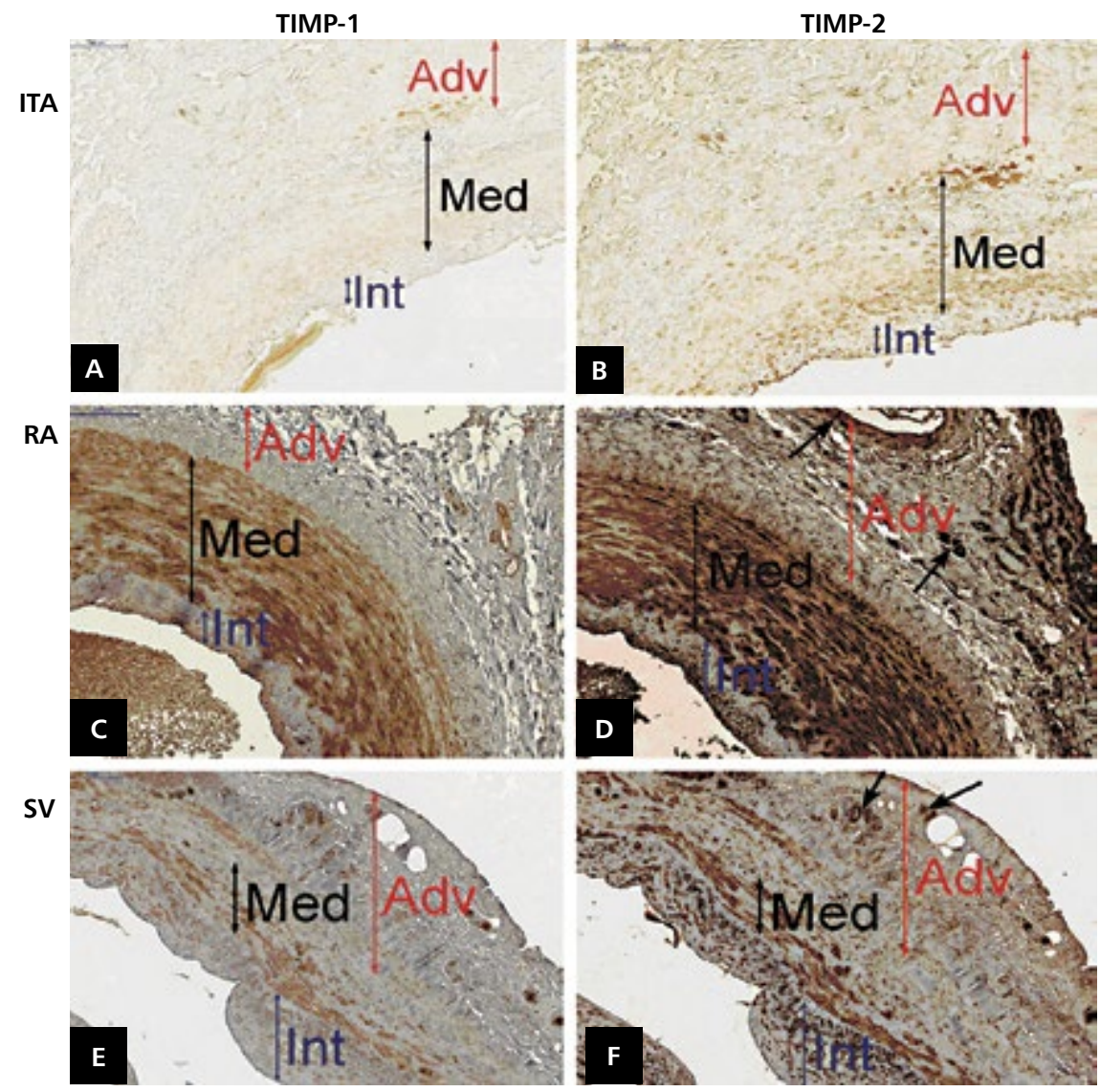

Figure 2. Expression of tissue inhibitors of metalloproteinases (TIMPs). The weakest immunostaining for TIMP-1 (A, C, E) and TIMP-2 (B, D, F) was seen in the tunica media (Med) of the internal thoracic artery (ITA) cross-sections; moderate, in saphenous vein (SV) wall; and the strongest, in radial artery (RA) segments. TIMP-2-positive cells were also found in the wall of the vasa vasorum (arrows) on RA and SV cross-sections; Adv — tunica adventitia; Int — tunica intima 
pared with approximately $10 \%(n=3)$ of RA and $15 \%$ $(n=15)$ of SV segments. A detailed analysis revealed that the MMP-2-positive ITA specimens were harvested from younger patients than the MMP-2-negative vessels (mean age, $62.2 \pm 11.6$ years vs. $66.8 \pm 5.9$ years; $p=0.001$ ). Contrary to the arterial segments, the MMP-2-positive SV segments were obtained from older patients (mean age, $72.3 \pm 10.3$ years) than the MMP-2-negative veins (mean age, $58.9 \pm 6.6$ years; $p<0.001$ ), although the latter group was relatively small (only 15 patients). Regarding MMP-9, immunostaining was positive in all RA and SV segments, as compared to less than $60 \%(n=59)$ of the ITA cross-sections. The MMP-9-negative ITA segments were obtained from younger patients than the MMP-9-positive segments (mean age, $60.7 \pm 10.6$ vs. $66.1 \pm 9.1$ years; $p=0.001$ )

Tissue inhibitors of metalloproteinase expression The weakest TIMP-1 and TIMP-2 expression was observed for the ITA; moderate, for the SV; and the strongest, for the RA (Table 2, Fig. 2). Moreover, the ITA was the only vessel type for which neither TIMP-1 $(\mathrm{n}=34 ; 33.7 \%)$ nor TIMP-2 $(\mathrm{n}=10$; $9.9 \%)$ immunoreactivity was seen on some cross-sections (IRS 0-1). The difference in global tissue expression of both TIMP-1 and TIMP-2 on the SV and RA cross-sections probably resulted from a stronger TIMP expression in the tunica media of the RA ( $p<0.001$; RA vs. SV).

\section{Balance of MMP and TIMP expressions}

The immunohistochemical analysis revealed a stronger expression of MMPs than TIMPs only in $30 \%$ of the arterial (both ITA and RA) cross-sections. In the majority of the SV segments, MMP tissue expression was comparable to or stronger than that of TIMPs. The only exception was the MMP-9-to-TIMP-2 ratio. The results are presented in Table 2.

\section{Late survival and adverse cardiac events}

Six (5.9\%) individuals were lost to follow-up (mean follow-up duration, $7.4 \pm 2.7$ years). Death was reported for 17 patients, including 10 patients who died due to cardiac reasons (acute coronary syndrome [ $\mathrm{n}=7$ ] or progressive left ventricular heart failure [ $\mathrm{n}=3]$ ). The mean probability of $12-, 36-$, and $60-$ month survival was $95.0 \% \pm 2.2 \%, 90.0 \% \pm 2.9 \%$, and $84.9 \% \pm 3.6 \%$, respectively. Moreover, in 20 patients, symptomatic CAD progression was noted. The mean survival rate without $\mathrm{CAD}$ progression, estimated according to the Kaplan-Meier curve, was $91.1 \% \pm 2.8 \%, 78.2 \% \pm 4.1 \%$, and $67.3 \% \pm 4.7 \%$, at 12,36 , and 60 months, respectively (Fig. 3).

\section{Patients with $C A D$ progression}

Based on the clinical status, 30 patients underwent emergent or elective coronary angiography. Additionally, in 20 asymptomatic patients, coronary angiography was performed but no progression of CAD was observed. Only in five patients,

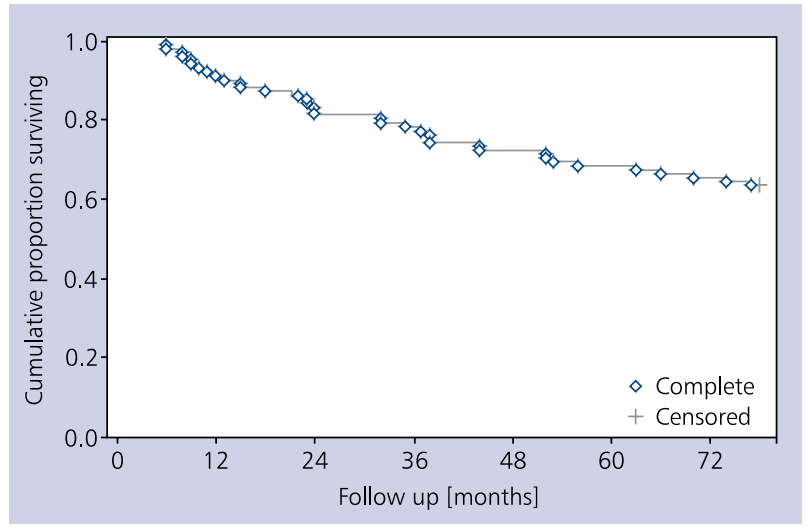

Figure 3. Coronary artery disease progression-free survival

Table 3. Results of follow-up coronary angiography

\begin{tabular}{lc} 
Significant lesions & Follow-up coronary angiography \\
No CAD progression & 20 \\
Native coronary arteries & 5 \\
SV & 21 \\
SV + RA & 2 \\
SV + RA + ITA & 2 \\
RA & 1 \\
\hline
\end{tabular}

Data are expressed as the number of patients. CAD — coronary artery disease; other abbreviations - see Table 2

CAD progression was observed in the native coronary arteries, which had not been treated during the primary surgery, resulting in clinical deterioration. In the remaining patients $(n=25)$, significant graft occlusions were observed, particularly in the SV segments ( $n=24)$, followed by the RA $(n=4)$ and ITA $(n=2)$ grafts. Detailed results of the follow-up coronary angiography are shown in Table 3.

Due to a small number of the arterial conduits with significant lesions at the end of follow-up, only histological parameters of the SV conduits were compared. In the vessel segments harvested from SVGD $(+)$ individuals, a markedly stronger tissue expression of both MMPs and a comparable TIMP expression was observed as compared with the SVGD(-) group (Table 4).

\section{DISCUSSION}

In this study we showed the variability in the expression of gelatinases (MMP-2 and MMP-9) and their inhibitors (TIMP-1 and TIMP-2) in the walls of vessels routinely used by cardiac surgeons as aortocoronary conduits. Moreover, we observed that tissue expression of MMPs is usually better balanced by TIMPs in the arteries than in the veins. These findings may have clinical significance and might have an impact on the long-term outcomes of the implanted grafts. 
Table 4. Comparison of matrix metalloproteinase (MMP) and tissue inhibitor of metalloproteinase (TIMP) tissue expression in saphenous vein (SV) grafts between patients with and without saphenous vein graft disease (SVGD)

\begin{tabular}{lccc|} 
Variable & $\begin{array}{c}\text { SVGD(+) } \\
(\mathbf{n}=\mathbf{2 4})\end{array}$ & $\begin{array}{c}\text { SVGD(-) } \\
(\mathbf{n}=\mathbf{7 2})\end{array}$ & $\mathbf{p}$ \\
\hline MMP-2 [IRS] & $6(6 ; 6)$ & $4(2 ; 4)$ & $<0.001$ \\
MMP-9 [IRS] & $4(4 ; 4)$ & $3(2 ; 4)$ & 0.005 \\
TIMP-1 [IRS] & $2(2 ; 4)$ & $2(2 ; 4)$ & 0.121 \\
TIMP-2 [IRS] & $6(6 ; 6)$ & $6(4 ; 6)$ & 0.139 \\
MMP-2/TIMP-1 & $2.0(1.5 ; 3.0)$ & $1.0(0.5 ; 2.0)$ & $<0.001$ \\
MMP-2/TIMP-2 & $1.0(1.0 ; 1.0)$ & $0.7(0.3 ; 1.0)$ & $<0.001$ \\
MMP-9/TIMP-1 & $2.0(1.5 ; 2.0)$ & $1.0(1.0 ; 2.0)$ & 0.001 \\
MMP-9/TIMP-2 & $0.7(0.7 ; 1.0)$ & $0.7(0.5 ; 1.0)$ & 0.613 \\
\hline
\end{tabular}

Variables are presented as medians ( $25^{\text {th }} ; 75^{\text {th }}$ percentile).

IRS - immunoreactivity score according to Remmele method

This report is part of a larger project aimed at supporting cardiac surgeons in decision making as to the optimal choice of vessels to bypass stenotic or occluded coronary artery segments. Although the effect of the demographic and clinical variables such as age, sex, quality of the native coronary arteries, or concomitant disorders on long-term outcomes of CABG has been examined for many years, we are convinced that a careful selection of conduits based on their biological properties is particularly important for procedural success [14, 15].

To our knowledge, we are the first to show that the preprocedural tissue expression of gelatinases was the weakest in the ITA walls compared with the other vessels, either arterial (RA) or venous (SV), which is one of the key histological findings in this study. Additionally, MMP-negative cross-sections were observed more frequently for the ITA than for the RA or SV. Gelatinases play a major role in the migration and proliferation of vascular smooth muscle cells, the key processes in neointima formation and atherosclerosis. Before migration of vascular smooth muscle cells, the surrounding ECM scaffold must be degraded by MMPs [16]. The gelatinases MMP-2 and MMP-9 have a unique ability to degrade elastin and collagen, the main components of the ECM [17]. Thus, they enable cell migration from the tunica media to the tunica intima through the internal elastic lamina. This leads to neointima formation that precedes the development of atherosclerosis. These pathological processes are observed both in damaged arterial walls (for example, after angioplasty for stenosis treatment) and in venous grafts implanted into arterial circulation $[12,18]$. This leads to complete occlusion and clinical disease recurrence. Thus, our finding indicating a stronger expression of MMPs in the RA or SV than in the ITA wall may explain the lowest rate of ITA graft occlusion during follow-up and, consequently, the most favourable long-term outcomes [4].
Our study also showed significant differences in TIMP expression in the walls of vascular segments. Under physiological conditions, a proteolytic activity of MMPs is strictly controlled by endogenous TIMPs [19]. Interestingly, we observed the lowest TIMP activity in the ITA, moderate in the SV, and the highest in the RA cross-sections. A previous study describing the effects of antiproliferative strategies reported a higher TIMP expression in successfully treated vessels [18]. Thus, the stronger immunostaining for TIMPs in the RA wall cells than in the SV sections observed in our study seems to have positive implications. It may at least partially explain why the use of RA grafts for CABG results in a lower rate of adverse cardiac events and a higher rate of patency in long-term follow-up than SV segments [3]. However, the marked differences in TIMP expression between grafts may raise some doubts as to the use of ITA segments. We believe that it is more important to analyse the balance of MMP-to-TIMP tissue expression than to assess MMP or TIMP activity separately, because the balance between MMP and TIMP expression has been found to be involved in maintaining normal structure and function of the vessels. This tissue remodelling, involving MMPs and TIMPs, has been considered a normal dynamic biological process [20]. On the other hand, an imbalance between MMP or TIMP activity was noted to be a factor underlying a number of vascular pathologies $[19,21]$. In our study, the balance between MMP and TIMP expression differed between the vessels used as aortocoronary bypass grafts. Although tissue expression of both TIMPs in the ITA cross-sections was the weakest, it was probably high enough to balance the minimal MMP activity. More importantly, tissue expression of MMPs calculated according to the IRS was usually weaker than that of TIMPs, or was at least balanced in the ITA cross-sections. The same was observed for RA segments but the absolute value of MMP and TIMP expression was stronger than that in the ITA wall. Contrary to the arterial grafts, the SV cross-sections showed a similar MMP expression but reduced TIMP activity compared with the RA, suggesting an imbalance that favoured MMP expression. It may at least partially explain why the long-term outcomes of the ITA are generally better compared with the other grafts and why the RA seems to be favoured over the SV [22, 23].

In our study, we also analysed long-term outcomes of CABG. We confirmed the previous findings showing that clinical deterioration after surgery is determined by unfavourable changes in aortocoronary grafts, predominantly in the SV segments [2]. In our group, in 21 of the 30 (70\%) patients with CAD progression after CABG, significant lesions in the $S V$ grafts were responsible for clinical deterioration. Moreover, the comparison of the SVGD(+) and SVGD(-) groups revealed the importance of the absolute value of MMP expression and the imbalance between MMP and TIMP activity. Thus, our study provides some additional data on the tissue and cellular levels, supporting recommendations for CABG that promote arterial revascularisation [24]. 
Our study has several limitations. Frist, not all patients who were included in the study underwent control coronary angiography. However, it is unlikely that non-diabetic patients without clinical progression of CAD had significant stenosis or occlusions in aortocoronary grafts. Such findings were previously reported by our team [12, 25]. Favourable long-term outcomes of arterial grafts, particularly the ITA, make it difficult to define the predictors of premature graft disease and failure. Such an analysis, although on a small number of vascular segments, was possible only for venous grafts. Thus, our conclusions need to be supported by studies involving more patients, particularly with occlusions in aortocoronary conduits. Moreover, the immunohistological analysis of the protein expression was performed soon after harvesting and before implantation into aortocoronary circulation. Ideally, such an analysis would be performed on the grafts explanted after a given period of time. However, this would be possible only in experimental animal models.

Another limitation is the fact that the ITA is exceptional in that it is elastic in its proximal and medial portions, less elastic close to its bifurcation, and its distal branches are fully muscular. More importantly, the internal elastic membrane loses its dense structure in the distal portion, becoming more permeable to infiltrating cells and less resistant to atherosclerotic degeneration [26]. The same changes may occur a result of age-related biophysical remodelling [27]. Thus, the distal part of the ITA should not be treated as representative for the whole length of the artery. Finally, tissue expression was assessed on the basis of immunohistochemistry. Although this method is accepted as a scientific tool to evaluate protein expression, it is qualitative rather than quantitative. While the IRS improves the quality of the analysis, a high level of scientific expertise is still needed. Involved in this project were histologists with considerable expertise, substantiated by a strong track record of peer-reviewed publications [8, 12].

In conclusion, gelatinase expression varies between the vessels used as aortocoronary conduits. An imbalance in the local haemostasis, favouring metalloproteinases over their tissue inhibitors in the wall of vessels before their application in patients undergoing $\mathrm{CABG}$, might predispose to earlier graft failure. Higher expression of TIMPs compared with gelatinases in arterial grafts might explain their favourable long-term outcomes. However, further studies on a larger group of patients with occlusions in aortocoronary grafts and with long-term follow-up are needed to confirm our primary results.

Funding: This study was supported by the State Committee for Scientific Research (Grant No. 5958/B/P01/2010/38; to A.M.). The funder had no role in study design, data collection and analysis, decision to publish, or preparation of the manuscript.

Conflict of interest: none declared

\section{References}

1. Banning A, Westaby S, Morice MC, et al. Diabetic and Nondiabetic Patients With Left Main and/or 3-Vessel Coronary Artery Disease. J Am Coll Cardiol. 2010; 55(11): 1067-1075, doi: 10.1016/j.jacc.2009.09.057.

2. Shavadia J, Norris CM, Graham MM, et al. Symptomatic graft failure and impact on clinical outcome after coronary artery bypass grafting surgery: Results from the Alberta Provincial Project for Outcome Assessment in Coronary Heart Disease registry. Am Heart J. 2015; 169(6): 833-840, doi: 10.1016/j.ahj.2015.02.022, indexed in Pubmed: 26027621.

3. Gaudino M, Benedetto U, Fremes S, et al. Radial-artery or saphenous-vein grafts in coronary-artery bypass surgery. $\mathrm{N} \mathrm{Engl}$ J Med. 2018; 378(22): 2069-2077, doi: 10.1056/NEJMoa1716026, indexed in Pubmed: 29708851.

4. Gansera B, Schmidtler F, Angelis I, et al. Patency of internal thoracic artery compared to vein grafts - postoperative angiographic findings in 1189 symptomatic patients in 12 years. Thorac Cardiovasc Surg. 2007; 55(7): 412-417, doi: 10.1055/s-2007-965372, indexed in Pubmed: 17902061.

5. Perek B, Casadei V, Puślecki M, et al. Clinical presentation, surgical management, and outcomes of patients treated for aortic stenosis and coronary artery disease. Does age matter? Kardiol Pol. 2018; 76(3): 655-661, doi: 10.5603/KP.2018.0005, indexed in Pubmed: 29313564.

6. Cao C, Manganas C, Horton M, et al. Angiographic outcomes of radial artery versus saphenous vein in coronary artery bypass graft surgery: a meta-analysis of randomized controlled trials. J Thorac Cardiovasc Surg. 2013; 146(2): 255-261, doi: 10.1016/j. jtcvs.2012.07.014, indexed in Pubmed: 22871565.

7. Perek B, Malinska A, Stefaniak S, et al. Predictive factors of late venous aortocoronary graft failure: ultrastructural studies. PLoS One. 2013; 8(8): e70628, doi: 10.1371/journal.pone.0070628, indexed in Pubmed: 23940610.

8. Podemska-Jedrzejczak Z, Malinska A, Sujka-Kordowska P, et al. Vascular restenosis in coronary artery bypass grafting might be associated with VEGF-C/VEGFR-3 signaling pathway. Heart Vessels. 2018; 33(9): 1106-1120, doi: 10.1007/s00380-018-1158-9, indexed in Pubmed: 29557990.

9. Thomas AC, Newby AC. Effect of matrix metalloproteinase-9 knockout on vein graft remodelling in mice. J Vasc Res. 2010; 47(4): 299-308, doi: 10.1159/000265564, indexed in Pubmed: 20016204.

10. Newby AC. Dual role of matrix metalloproteinases (matrixins) in intimal thickening and atherosclerotic plaque rupture. Physiol Rev. 2005; 85(1): 1-31, doi: 10.1152/physrev.00048.2003, indexed in Pubmed: 15618476.

11. Wang Xi, Khalil RA. Matrix metalloproteinases, vascular remodeling, and vascular disease. Adv Pharmacol. 2018; 81: 241-330, doi: 10.1016/bs.apha.2017.08.002, indexed in Pubmed: 29310800.

12. Perek B, Malinska A, Misterski M, et al. Preexisting high expression of matrix metalloproteinase-2 in tunica media of saphenous vein conduits is associated with unfavorable long-term outcomes after coronary artery bypass grafting. Biomed Res Int. 2013; 2013: 730721, doi: 10.1155/2013/730721, indexed in Pubmed: 24151618.

13. Remmele W, Stegner HE. [Recommendation for uniform definition of an immunoreactive score (IRS) for immunohistochemical estrogen receptor detection (ER-ICA) in breast cancer tissue]. Pathologe. 1987; 8(3): 138-140, indexed in Pubmed: 3303008.

14. Mohr F, Morice MC, Kappetein A, et al. Coronary artery bypass graft surgery versus percutaneous coronary intervention in patients with three-vessel disease and left main coronary 
disease: 5-year follow-up of the randomised, clinical SYNTAX trial. Lancet. 2013; 381(9867): 629-638, doi: 10.1016/s01406736(13)60141-5.

15. Trzeciak P, Karolak W, Gąsior M, et al. In-hospital and long-term outcomes of coronary artery bypass graft surgery in patients $\leq 45$ years of age and older (from the KROK registry). Kardiol Pol. 2017; 75(9): 884-892, doi: 10.5603/KP.a2017.0090, indexed in Pubmed: 28553871.

16. Johnson JL, van Eys GJ, Angelini GD, et al. Injury induces dedifferentiation of smooth muscle cells and increased matrix-degrading metalloproteinase activity in human saphenous vein. Arterioscler Thromb Vasc Biol. 2001; 21(7): 1146-1151, indexed in Pubmed: 11451743.

17. Crowther M, Goodall S, Jones JL, et al. Increased matrix metalloproteinase 2 expression in vascular smooth muscle cells cultured from abdominal aortic aneurysms. J Vasc Surg. 2000; 32(3): 575-583, doi: 10.1067/mva.2000.108010, indexed in Pubmed: 10957667.

18. Wu Z, Zang S, Liu W, et al. Cryoplasty for canine iliac artery stenosis and its impact on expression of TIMP-2 and MMP-2. Vasc Endovascular Surg. 2015; 49(5-6): 135-141, doi: 10.1177/1538574415603488, indexed in Pubmed: 26335992.

19. Raffetto J, Khalil R. Matrix metalloproteinases and their inhibitors in vascular remodeling and vascular disease. Biochem Pharmacol. 2008; 75(2): 346-359, doi: 10.1016/j.bcp.2007.07.004.

20. Galis ZS, Khatri JJ. Matrix metalloproteinases in vascular remodeling and atherogenesis: the good, the bad, and the ugly. Circ Res. 2002; 90(3): 251-262, indexed in Pubmed: 11861412.

21. Aravind B, Saunders B, Navin T, et al. Inhibitory effect of TIMP influences the morphology of varicose veins. Eur J Vasc Endo- vasc Surg. 2010; 40(6): 754-765, doi: 10.1016/j.ejvs.2010.04.028, indexed in Pubmed: 20598922.

22. Zhu Y, Chen A, Wang Z, et al. Ten-year real-life effectiveness of coronary artery bypass using radial artery or great saphenous vein grafts in a single centre Chinese hospital. Interact Cardiovasc Thorac Surg. 2017; 25(4): 559-564, doi: 10.1093/icvts/ivx174, indexed in Pubmed: 28637292.

23. Schwann TA, Hashim SW, Badour S, et al. Equipoise between radial artery and right internal thoracic artery as the second arterial conduit in left internal thoracic artery-based coronary artery bypass graft surgery: a multi-institutional study+. Eur J Cardiothorac Surg. 2016; 49(1): 188-195, doi: 10.1093/ejcts/ezv093, indexed in Pubmed: 25762396.

24. Kieser TM, Curran HJ, Rose MS, et al. Arterial grafts balance survival between incomplete and complete revascularization: a series of 1000 consecutive coronary artery bypass graft patients with 98\% arterial grafts. J Thorac Cardiovasc Surg. 2014; 147(1): 75-83, doi: 10.1016/j.jtcvs.2013.08.003, indexed in Pubmed: 24084283.

25. Perek B, Malińska A, Ostalska-Nowicka D, et al. Cytokeratin 8 in venous grafts: a factor of unfavorable long-term prognosis in coronary artery bypass grafting patients. Cardiol J. 2013; 20(6): 583-591, doi: 10.5603/CJ.2013.0142, indexed in Pubmed: 24338534.

26. Borović ML, Borović S, Perić M, et al. The internal thoracic artery as a transitional type of artery: a morphological and morphometric study. Histol Histopathol. 2010; 25(5): 561-576, doi: 10.14670/HH-25.561, indexed in Pubmed: 20238295.

27. Perek B, Kowalska K, Kempisty B, et al. Gender and age-related variability of macrophage representation in the internal thoracic artery wall: does it matter? J Biol Regul Homeost Agents. 2018; 32(4): 791-802, indexed in Pubmed: 30043561.

Cite this article as: Perek B, Kowalska K, Nowicki M, et al. Variability in gelatinase expression in the walls of vessels used as aortocoronary conduits may impact long-term graft patency. Kardiol Pol. 2019; 77(2): 217-224, doi: 10.5603/KP.a2019.0015.

\section{WHAT IS NEW?}

The left internal thoracic artery graft to the left anterior descending artery has been considered a gold standard for many years. In the meantime, there has been an ongoing debate about which grafts should be sutured to the other arteries. More and more evidence shows that the radial artery might be better than the saphenous vein regarding long-term outcomes. Our study revealed that one of the possible explanations of this phenomenon might be an imbalance between the expression of matrix metalloproteinases and their tissue inhibitors in the walls of grafts (harvested from the same patients) before coronary artery bypass grafting. We showed that this imbalance was more prevalent in the venous than in the arterial wall cross-sections. Moreover, the results of the immunohistochemical analysis of protein expression in the graft walls were related to the long-term clinical outcomes of coronary artery bypass grafting. 